\title{
The BodyMind Approach ${ }^{\mathrm{TM}}$ and people affected by medically unexplained symptoms /somatic symptom disorder
}

\author{
Helen Payne
}

\section{Introduction}

This chapter outlines a new and novel clinical service in primary care in National Health Service (NHS) England for patients with persistent bodily symptoms (such as fibromyalgia, irritable bowel syndrome (IBS), chronic fatigue, chronic pain, non-cardiac chest pain, dizziness, skin conditions etc.) for which tests and scans come back negative, termed medically unexplained symptoms (MUS). The service is based on preliminary research (Payne and Stott, 2010) and several pilots (Payne, 2014; Lin and Payne, 2014; Payne and Brooks, 2016; Payne and Brooks, 2017) of delivery with positive outcomes for patients and savings to the NHS. The intervention is termed The BodyMind Approach ${ }^{\mathrm{TM}}$ (TBMA) which employs an animated (Sheets-Johnstone, 2016) methodology emanating from dance movement psychotherapy and the recent neuroscience research (Gallese, 2011; Shore, 2012) integrating body and mind (physical and mental health).

Participants are General Physician (GP) or self-referred to 12 session groups with one-to-one meetings with the group facilitators prior to, and following, the group experience. Phase two engages patients in non-face-to-face contact over six months to embed their self-designed action plan based on their new perceptions following the group process. Assessments for anxiety, depression, symptom distress, functioning/activity and wellbeing are conducted pre, post and at six months' follow up. GP and hospital visits are monitored together with social support, employment and leisure pursuits.

The findings from the delivery of TBMA as an MUS Clinic in primary care thus far have mirrored the original research outcomes including that participants reduce in their scores for symptom distress, anxiety and depression. Furthermore, they increase in activity/functioning and wellbeing and were able to self-manage their symptoms far better as a result. It can be cautiously concluded that this intervention may offer a useful solution to the vast numbers of patients in primary care with MUS for whom cognitive behaviour therapy (CBT) has been rejected or tried and found unsatisfying.

\section{Medically unexplained symptoms}

MUS are a long term condition and a global problem with very high health utilisation resulting in large costs and wastage. Patients with MUS account for an estimated 15-30\% of all primary care consultations (Kirmayer et al., 2004) and GPs report that these can be among the most challenging consultations they provide. Other studies in primary care show $19-50 \%$ visits are for MUS (Reid et al., 2002; Preveler, 1997; Barsky and Borus, 1995). MUS account for a more than $20 \%$ of all outpatient activity among frequent attenders (Reid et al., 2001), mainly in neurology, gastroenterology, rheumatology, cardiology, gynaecology (Jackson et al., 2006; Burton, 2003; Nimnuan et al., 2001; Kooiman et al., 2000; Hamilton et al., 1996). MUS is the fourth most costly population in primary care and the most costly diagnostic category of out-patients. The cost in 2009 was over $£ 3$ billion per year rising to $£ 18$ billion if quality of life, benefits and absence from work are included (Bermingham et al., 2010).

There is a wide range of symptoms falling into MUS and there are no effective treatments except pain relief medication for some, and, for IBS and chronic fatigue, CBT, which has been found to be no more effective than routine care for generic MUS (Sumathipala, 2008). Less than $10 \%$ of MUS 
sufferers receive anti-depressant treatment or accept psychological therapy leaving the majority having to "learn to live with it" as has so often been said to them (Hamilton et al.,1996; Fink, 2002; Mangwana et al., 2009; Hansen et al., 2001). Some GPs are rather good at working with such patients but many are not, feeling frustrated and at a loss.

Consequently, the author, after noticing this large population without support or hope for change, conducted a four year research programme to explore whether an embodied group methodology might offer support for this large and varied patient population in the NHS. Subsequently, and following market research and cost effectiveness studies, a spin-out clinic from within The University of Hertfordshire was funded called Pathways2Wellbeing. This service is based on biopsychosocial and recovery models designed specifically for people with MUS, some of whom have fearful attachment and /or trauma. It is delivered as a sort of pre-therapy, starting at where the patient is, i.e. with their bodily distress. The patient's relationship with the symptom is worked with by employing practices derived and adapted to this patient group from the arts, bodymindfulness and dance movement psychotherapy. The initial focus is on creating distance between the symptom and the patient through symbolic and metaphoric practices, for which the arts are a vehicle, enables a strong sense of safety and holding for the unconscious, and unknown knowns about the symptom to emerge into consciousness.

It should be noted that in 2013 the DSM 5 was introduced (American Diagnostic and Statistical Manual of Mental Disorders, 2013) in which somatoform disorders were replaced by the category Somatic Symptom Disorder (SSD), consisting of a new set of criteria including positive psychological ones. For the diagnosis of a SSD the presence of MUS is no longer a criterion. Among the reasons for this decision are patient and GP dislike of the term MUS, the low inter-rater reliability and questionable validity of physicians' judgments on whether somatic symptoms are medically explained or not (Fink, Rosendal and Olesen, 2005) and the fact that many symptoms change back and forth between being considered medically explained or unexplained (Klaus, Rief, Brähler, Martin, Glaesmer and Mewes, 2013). Therefore the 'A' criterion includes the presence of at least one very disabling or distressing physical symptom (explained or unexplained). The ' $\mathrm{B}$ criteria' includes several psychological features such as, health anxiety (Barsky and Borus, 1995), disproportionate and persistent thoughts about one's symptoms (Verhaak, Meijer, Visser and Wolters, 2006), and excessive time and energy devoted to these symptoms or health concerns (Jackson and Passamonti, 2005). Consequently, the indicator of severity is no longer based on the number of somatic symptoms, but on the severity of psychological features specified under the ' $B$ ' criteria. The ' $C$ ' criterion addresses chronicity i.e. typically a duration of symptoms of at least six months.

These new criteria have been criticized. They were described as clinically unhelpful (Mayou, 2014). Furthermore, they seem to cover an extremely heterogeneous group of patients (Rief and Martin, 2014). Furthermore, research (Khan, Khan, Harezlak, Tu, and Kroenke, 2003) has shown that rarely are doctors incorrect with their diagnosis $(0.69 \%)$. The members of the DSM somatic symptoms workgroup stated that the usefulness and reliability of the specific criteria still remains to be determined (Dimsdale, Creed, Escobar, et al., 2013). Consequently, the author continues to employ the tem medically unexplained symptoms for the time being.

\section{The BodyMind Approach}

The clinic being delivered by Pathways 2 wellbeing in the primary care section of the health service in England employs a research-informed group work methodology termed The BodyMind Approach $^{\mathrm{TM}}$ (TBMA). It offers experiential learning through exercises involving the body bearing the symptom/s in mind. Over time participants make mindful and meaningful connections. The name arose from interviews with patients (co-researchers), in an early research study, who referred to their 
experience of the group process as enabling them to make significant associations between their bodies and their minds.

John Dewey (Davidson, 2004 p198), the philosopher, identified that there were deeply connected paths of knowing, where the body and mind overlap becoming entwined, he termed this the 'BodyMind' (Dewey, 1988 p191) 'it is the movement which is primary, and the sensation which is secondary, the movement of body, head and eye muscles determining the quality of what is experienced' (Dewey, 1896 p358). Dewey used a hyphen between the two words, this author has joined them together to form the term BodyMind to represent that the two are not distinct but complementary aspects. The capitalised $\mathrm{M}$ in Mind reminds us of the equal importance of the mind with the body and the term capitalises on the inter-relationship between the two. Body is written first in the term, as opposed to the usually written 'mind-body' since it is the body which is the focus in this methodology. Through addressing the sensory experience in the lived body the mind becomes apparent.

For the purposes of this chapter the definition of embodiment is both the recognition that cognitive processes are embedded in the brain and that the origin of these processes are in the sensory-motor experience. Acting and perceiving are therefore not understood as a physical-mental dichotomy, but as interlinked (Fuchs, 2009). The lived body (i.e. the subjective body) has an impact on the mind, consequentially a focus on experiencing the sensory body and its action can be employed to help change the minds of patients.

The emphasis in TBMA is on nurturing a re-association with the body (and the symptom/s), engaging with a new perception and thus a different lived body experience. It acknowledges the interaction between the inter-corporeal aspects of the knowing body. It has a focus on the changing and always embodied present moment which allows for the experiential sensation of the symptom to be addressed in unique and novel ways. Because TBMA works directly at the site where much of the distress and damage occurs, the lived body, TBMA trained facilitators are uniquely positioned to address the embodied consequences of persistent unexplainable bodily symptoms on the patients' lived body experience. By helping patients to accept and learn from their symptom they become empowered to feel less restricted in their lives despite the symptom. TBMA is not a cure but a methodology to help patients to learn to live well with their symptoms. Having said this there are some patients who do fully recover from their symptoms. It employs metaphors and experiential exercises in response to negative sensory symptoms for example pain or discomfort. There is an exploration of personal values whereby goals and practices are arrived at to improve overall quality of life in an action plan drawn up towards the end of the course. Furthermore, it is particularly accessible to the many MUS patients who reject CBT.

Some patients report that they cannot feel their bodies, i.e. sensations from the body are only experienced as fatigue or pain, for example (Payne, 2015; 2016). There is a lack of experiential joining of body signals with the sense of being moved by the world around them. Somatic autonomous processing of experiential content such as the quickening of the heartbeat or minimal breathing in the upper chest become isolated into somatic pathways and reported as medical symptoms. Many patients have overwhelming concerns about their somatic wellbeing, but are frequently unable to connect this content to their current or past life situations. TBMA enables patients to explore their symptoms safely leading to meaning-making and connections with life experience e.g. trauma /attachment issues.

TBMA reduces overall distress as well as the patient's preoccupation with MUS by teaching coping strategies to begin with, for example, progressive muscle relaxation, diaphragmatic breathing, activity regulation, kind attention to, and caring of, the body, somatic and emotional awareness increasing adaptive interpretations of the self and the world. Employing imagery and visualization can reduce bodily tension providing a different experience of the body. Activity regulation can involve problem 
solving rather than avoidance or withdrawal as habitual reactions to adversity. Self-monitoring and bodymindfulness help patients increase somatic and emotional awareness. Through employing the arts such as mark-making, clay, creative movement and writing patients learn to change interpretations of the self and the world (e.g. feelings of being overwhelmed, all-or-nothing attributions or catastrophizing). Since it is a facilitated group setting they also learn about communication such as interpersonal processes and assertiveness. Personalized action plans, enacted following the group experience, arise from the new learning and psycho-education. They focus on embedding different behaviours and coping strategies which help to minimize discomfort and increase functioning despite the symptoms.

TBMA facilitators nurture the development of narratives that connect somatic symptoms with psychosocial factors and where possible with past experiences and patients' cultural beliefs. Due to the complexity of medical and psychosocial presentations, it is important to help patients understand how everything fits in. The arts, through symbol and metaphor, can help patients discover new explanatory models (rather than solely the preferred physical) empowering them to embrace selfmanagement through lifestyle changes, leisure pursuits, social activities, emotional regulation, relaxation, or stress management, as well as the kindly acceptance of symptoms. Cultivating alternative narratives tailored to patients' specific symptoms and culturally-based beliefs helps in alleviating the distress associated with living with the unknown i.e. having no explanation for the persistent symptoms.

The TBMA model reassures patients that their symptoms are real diffusing the emotional charge, and helping patients feel understood and believed. The non-judgmental, accepting group culture assists patients in letting go of the tendency to convince others of the legitimacy of their symptoms.

By addressing the subjective body experience whereby emotion, sensory-motor-body experience, cognition, action, and perception are unified, change in self-experience, emotions and behaviour is possible and resilience promoted. Emotion and cognition are mirrored in movement and body posture, however, they are also influenced by them (Koch and Fuchs, 2011). We can all feel in our bodies how, by expressing an emotion such as 'sadness' in our posture, for example, retreating and sinking, head bowed, hands to upper body, collapsing in the chest, a change in self experience results (perhaps you, dear reader, might try this posture now to see what feelings arise). Furthermore, according to Carney et al., (2010) dominant versus submissive postures can even change testosterone levels, saliva secretion and risk-taking behaviour. Emotion and movement/posture are inextricably connected. Embodied approaches emphasize the meaning of the sensorimotor experience for cognition, affect, and interaction, operating at the centre of emotional processing and self-regulation. Somatic sensations can become a vehicle for an unknown/unconscious, nonconscious attitude, trauma, or affect which has become detached from their motivational context (Sass, 2000). The consequence is that the patient is incapable of making sense of felt emotions as well as adequately expressing or following them, subsequently somatic symptom/s can emerge as a replacement for this expression. By integrating sensory and emotional awareness through corporal practices the range of affect, communicative and expressive behaviours can be expanded and symptoms explored safely.

TBMA integrates embodied approaches with mindfulness and behaviour change strategies offering a distinctly flexible and versatile intervention appealing to the whole person i.e. the cognitive, physical/kinaesthetic, sensory and emotional aspects. Consequently it can reach a wide range of patients and facilitators. By directing kind attention and mindfulness to the body and its connection to the self, practices in TBMA meet the basis of the symptom distress to change the subjective relationship with the symptom/body. The facilitated group setting promotes social attention, the group interactive practices encourage attention towards the bodily mediated emotions of others and their impact on the self. 


\section{Typical profile of a patient suffering medically unexplained symptoms}

Ms G is a 45-year-old woman, married, and is a mother of two. She, formerly worked as a teaching assistant and is, now on disability benefits. She initially presented six years previously with abdominal pain located in the right upper quadrant, gradual in onset, very severe in intensity, aching in character, and non-radiating. The pain was constant temporally with no remissions. She rated the pain as a 10/10 at its worst and a 7/10 on average. During the first visit, no aggravating, relieving, or precipitating factors were identified. Her abdominal pain was associated with mild headache aggravated by stress. There was no history of appetite or weight loss, dyspepsia, or alcohol abuse. Ms $\mathrm{G}$ had undergone appendectomy for acute abdominal pain ten years ago with little long-term relief. In the last two years she had referrals to two gastroenterologists, both reported negative findings after extensive investigations.

Ms G was not relieved by these findings continuing to be very distressed during GP visits. She complains of unwavering pain and how overwhelmed she feels with trying to manage her health and care for her family. Although she appears to experience some relief with reassurances that "there is nothing wrong with her physically" and even leaves some visits with a sense of boosted self-efficacy and optimism, she deteriorates during visits to the point of crying uncontrollably. Worrying statements such as, "I can't take this anymore. I don't know how I can go on living like this," result in the GP having to "talk her down from the ledge". It feels like nothing helps her in the long run.

The frequent visits to the GP often end with adjustments to medication or an agreement to discuss the value of continued investigations, imaging, and medical procedures. The GP breathes a sigh of relief each time she leaves but doubts the effectiveness of any agreed treatment or investigation. The GP is concerned about Ms G's mental health and believes there is a connection between her anxiety, frustration, and somatic symptoms. Ms G though vacillates on stress as a factor in the exacerbation and maintenance of her abdominal pain. She refuses referrals to psychological services, and since there is no pending psychiatric risk the GP has not insisted. Eventually, after six years the GP invites Ms G to consider a Pathways2Wellbeing group course for people suffering persistent physical symptoms for which tests and scans come back negative. She reassures Ms G that others have enjoyed the courses and found them beneficial so asks her "why not give it a try?" She asks lots of questions to which the GP can respond with reassuring answers. At last she agrees to follow through with this referral.

Ms $\mathrm{G}$ was given the leaflet about pathways 2 wellbeing, advertising the course, by her doctor. The doctor suggested she might try this clinic in the meantime. She came to her first individual meeting saying that she felt desperate and wanted to try this group as she thought it was something different from what she had been offered so far by the NHS. In the group from the very first session she showed a willingness to engage in whatever she was asked to do, although she found it a little challenging in view of her continuous abdominal pain. She missed two sessions out of 12 (sessions 3 and 5), in both cases she was in too much pain to be able to attend. However, in each attended session she showed an interest in others. She found it useful talking about her pain and also to listen to others sharing about their difficulty to cope with their painful body, their surrendering attitude, not being listened to properly and not being understood. These were common themes, participants were listened to well and could share their struggles in dealing with pain as they felt understood without judgment.

During the group process Ms G was invited by her facilitator to be kinder to herself, as, in her language there was a derogatory regard towards herself, but in session six she announced to the group that she was "a wonderful person". It meant a lot for her to say this to her group without feeling judged. She told the group that she was repeating this to herself and that it was convincing, she started to believe it. There was no self-blaming and no self-denigrating anymore. She told the group that she was starting to see her depression lifting. In the group, during the body movement practices she had 
the most sensitive perception and was able to capture in other people's movement, something about them. She started trusting herself more and her self-esteem visibly increased.

Week after week she was telling the group how she was much more relaxed, understanding and compassionate about her condition. Her emotional pain seemed to be located in her stomach, her voice was broken as she was talking but the awareness of her tension in her stomach seemed to soften her muscles and she felt better. All this took place in only ten sessions.

At the exit meeting she said that at the beginning of the course she did not know what to expect, was fearful and resistant and felt really down. The group helped her to feel less isolated, she learnt how to make use of the optimal diaphragmatic breathing that she had practiced in the group and this had helped to ease the pain in her abdomen for quite some time. She also learnt that the body movement helps to release her tension and lift her mood.

\section{Individual Monitoring}

Data was collected the week before the group began, the week after it ended and at 6 months follow up. The following tools were employed to collect the data at these time points:

\section{Measure Yourself Medical Outcome Profile Scores (MYMOP2)}

The MYMOP2 is an individualised patient rated outcome questionnaire. It is problem-specific and measures two symptoms chosen by the patient. It also includes general wellbeing and impact of symptoms on a chosen activity. The greater the score, the more severe the symptoms are.

\section{Primary Healthcare Depression Scale ( $P H Q$ 9)}

This is a client rated tool for depression. It scores each of the 9 depression DSM-IV criteria as " 0 " (not at all) to " 3 " (nearly every day). Depression severity is graded based on the PHQ-9 score 0-4 none; 5-9 mild; 10-14 moderate; 15-19 moderately severe; 20-27 severe.

\section{Generalised Anxiety Disorder (GAD 7)}

This is a client rated measure for assessing generalized anxiety disorder on a 7-item self-rating scale. It scores each item as " 0 " (not at all) to " 3 " (nearly every day) for each item. Severity of generalized anxiety is graded based on the GAD 7 score 0-4 none; 5-9 mild; 10-14 moderate; $15-21$ severe.

\section{The Global Assessment of Functioning Scale (GAF)}

This is a clinician rating tool used to measure overall level of psychological, social and occupational client functioning on a scale ranging from 1 to 100. The higher the score, the higher the level of functioning there is. GAF covers the range from positive mental health to severe psychopathology.

Further data is collected via a tailor-made questionnaire

For this patient her perceived social support pre-course was satisfactory, post course it was good. She continued with medication pre and post course.

With reference to psychological insight Question 1 asks "Do you believe the way you think and feel can affect your physical symptoms?" Question 2 asks "Do you believe that your physical symptoms can affect the wan you think and feel?" there was a strong belief in the mind body connection evident at post course and follow up. 
Her individual monitoring is shown in Table I below:

\section{Insert Table 1 about here}

From the scores it appears that Ms G has increased her activity from pre to post course, as well as her general functioning levels. This ties in with her perceived social support being better at post course than at pre course. This may be as a result of feeling a sense of belonging in the group thus reducing her isolation. Feeling less anxious and depressed might possibly be due to being able to endure the pair, tolerate it or it actually decreasing post course when compared to pre course. It is interesting that she strongly believes in the connection between mind and body following the experience of the course whereas before the course it was at a weaker level. Despite her wellbeing score remaining the same level pre to post course there is an indication that the pain has reduced and overall MYMOP scores reduced slightly.

\section{Conclusion}

There is some evidence that TBMA applied as group work in primary care for people affected by medically unexplained symptoms, albeit with small numbers so far, can be an effective way to help people self-care and live well with their symptoms. By gaining an understanding and self-compassion within a supportive group environment it seems this new perspective changes their way of being with their symptoms. There is more to be done to evidence the methodology however an encouraging start has been made.

\section{Acknowledgements}

Appreciations go to the participants, the team at Pathways2Wellbeing and to the University of Hertfordshire for the support they have provided.

\section{References}

American Psychiatric Association, Diagnostic and Statistical Manual of Mental Disorders, (2013) American Psychiatric Publishing, Washington, DC: Arlington, VA

Barsky, A \& Borus, J. (1995). Somatization and medicalization in the era of managed care. Journal of the. American Medical Association, 274, (24), 1931-1934.

Bermingham, S; Cohen, A; Hague, J and Parsonage, M. (2010). The cost of somatisation among the working-age population in England for 2008-09. Mental Health in Family Medicine, 7, 71-84.

Burton, C. (2003). Beyond somatisation: a review of the understanding and treatment of medically unexplained physical symptoms (MUPS). British Journal of General Practice, 53, 488, pp. 231-9

Carney, D. R., Cuddy, A. J. C., and Yap, A. J. (2010). Power posing: brief nonverbal displays affect neuroendocrine levels and risk tolerance. Psychological Science, 21, 1363-1368. doi: $10.1177 / 0956797610383437$

Clark, D. and Oates, M. (2014). Improving Access to Psychological Therapies: Measuring Improvement and Recovery in Adult Services, version 2. Accessed 21.6.16 www.iapt.nhs.uk/silo/files/measuring-recovery-2014.pdf

Davidson, D. (2004). Problems of rationality. New York: Oxford University Press.

Dewey, J. (1988). Experience and nature. In: JA Boydston (Ed.), John Dewey: the later works, 1925- 
1953: Vol 13, 1938-1939 (pp1-62) (original work published in 1938). Carbondale: Southern Illinois University Press.

Dewey, J. (1896). The Reflex Arc Concept in Psychology, Psychological Review, 3: 357-370.

Dimsdale, J.E; Creed, F; Escobar, J et al., (2013). Somatic symptom disorder: an important change in DSM. Psychosomatic Research, 75, (3), 223-228

Dugas, M.T. and Robichaud, M. (2007). Cognitive behavioural treatment for general anxiety disorder: From science to practice. London: Routledge.

Fink, P; Rosendal, M; and Toft, T (2002). Assessment \& treatment of functional disorders in general practice: the extended reattribution $\&$ management model-an advanced program for non-psychiatric doctors. Psychosomatics 43, 2, 93-131.

Fink, P; Rosendal, M; and Olesen, F. (2005). Classification of somatization and functional somatic symptoms in primary care. Australia and New Zealand Journal of Psychiatry; 39, (9), 772-781.

Gallese, V (2011) Neuroscience and phenomenology. Phenomenology and Mind, 1, 33-48.

Guyatt, G.H; Juniper, E.F; Walter, S; Griffith, L and Goldstein, R.S. (1998). Interpreting treatment effects in randomised trials. British Medical Journal, 1998; 316:690-693.

Gyani, A; Shafran, R; Layard, R; and Clark, D.M. (2013). Enhancing recovery rates: Lessons from year one of IAPT. Behaviour Research Therapy. Sep; 51(9): 597-606. doi: 10.1016/j.brat.2013.06.004

Hamilton, J., Campos, R. \& Creed, F. (1996). Anxiety, depression and management of medically unexplained symptoms in medical clinics. Journal of The Royal College of Physicians of London, 30, $18-20$.

Hansen, MS; Fink P; Frydenberg, M; Oxhoj, ML; Sondergaard, L; and Munk-Jorgensen, P. (2001). Mental disorders among internal medical inpatients: Prevalence, detection and treatment status. Psychosomatic Research, 50, 4, 169-204

Jackson, JL and Passamonti, M (2005). The outcomes among patients presenting in primary care with a physical symptom at 5 years. General International Medicine, 20, (11), 1032-1037.

Jackson, J; Fiddler, M; Kapor; Wells, A; Tomenson, B; and Creed, F. (2006). Number of bodily symptoms predicts outcome more accurately than health anxiety in patients attending neurology, cardiology \& gastroenterology clinics. Psychosomatic Research; 60, 4, 357-63.

Khan AA; Khan A; Harezlak J; Tu W; and Kroenke K. (2003). Somatic Symptoms in primary care: aetiology and outcome. Psychosomatics; 44:471-8.

Koch, S. C., and Fuchs, T. (2011). Embodied arts therapies. Arts in Psychotherapy, 38, 276-280. doi: 10.1016/j.aip.2011.08.007

Kooiman, CG; Bolk, JH; Brand, R Trijsburg, RW; and Rooijmans, HGM (2000) Is Alexithymia a Risk Factor for Unexplained Physical Symptoms in General Medical Outpatients? Psychosomatic Medicine; 62:768-778

Klaus, K; Rief, W; Brähler, E; Martin, A; Glaesmer, H; and Mewes, R. (2013). The distinction between "medically unexplained" and "medically explained" in the context of somatoform disorders. Int. J. Behavioural Medicine. 20; 161-171.

Kroenke, K \& Spitzer, RL (2002) Diagnostic and severity measure. Psychiatric Annals, 32, 9, Sept 2.

Mangwana, S; Burlinson, S; and Creed, F (2009). Medically unexplained symptoms presenting at secondary care--a comparison of white Europeans and people of South Asian ethnicity. Psychiatry in Medicine. 39; 1; 33-44

Mayou, R (2014). Is the DSM-5 chapter on somatic symptom disorder any better than DSMIV somatoform disorder? British Journal of Psychiatry, 204, (6), 418-419. 
Nimnuan, C., Hotopf, M., and Wessely, S. (2001). Medically unexplained symptoms - An epidemiological study in seven specialities. Psychosomatic Research, 51(1), 361-367. 10.1016/S0022-3999(01)00223-9

Payne, H. and Stott, D. (2010). Change in the moving bodymind: Quantitative results from a pilot study on the BodyMind Approach (BMA) as groupwork for patients with medically unexplained symptoms (MUS). Counselling and Psychotherapy Research, 10, 4, 295-307.

Lin, Y and Payne, H. (2014). The BodyMind Approach ${ }^{\mathrm{TM}}$, Medically Unexplained Symptoms and Personal Construct Psychology. Body, Movement and Dance in Psychotherapy, 9, 3.

Payne, H. (2014). The BodyMind Approach ${ }^{\mathrm{TM}}$ : the treatment of people with medically unexplained symptoms. The Psychotherapist, summer, issue 57, 30-32.

Payne, H. (2015). The Body speaks its Mind: The BodyMind Approach ${ }^{\mathrm{TM}}$ for patients with Medically Unexplained Symptoms in UK primary care. Arts in Psychotherapy, 42, 19-27.

Payne, H and Brooks, S. (2016). Clinical outcomes and cost benefits from The BodyMind Approach $^{\mathrm{TM}}$ for Patients with Medically Unexplained Symptoms in Primary Health Care in England: Practice-Based Evidence. Arts in Psychotherapy, 47, 55-65.

Payne, H. (2016). The BodyMind Approach ${ }^{\mathrm{TM}}$. Healthcare, Counselling and Psychotherapy Journal, October 2016, vol 16, issue 4, pp 14-18. British Association for Counselling and Psychotherapy,

Payne, H and Brooks, S. (2017). Moving on: A summary of practice-based evidence from The BodyMind Approach ${ }^{\mathrm{TM}}$ for patients with medically unexplained symptoms in primary care. Public Mental Health Journal (in press).

Peveler, R; Kilkenny, L and Kinmonth, A.L. (1997). Medically unexplained physical symptoms in primary care: A comparison of self-report screening questionnaires and clinical opinion. Psychosomatic Research; 42: 245-252.

Reid, S; Wessely, S; Crayford, T; and Hotopf, M. (2002). Frequent attenders with medically unexplained symptoms: Service use and costs in secondary care. British Journal of Psychiatry, 180:248-253.

Rief, W and Martin, A. (2014). How to use the new DSM-5 somatic symptom disorder diagnosis in research and practice: a critical evaluation and a proposal for modifications. Annual Review of Clinical Psychology, 10; 339-367.

Sass, L. A. (2000). Schizophrenia, self-experience, and so-called "negative symptoms" reflections on hyper-reflexivity. In D. Zahavi (Ed) Exploring the Self: Philosophical and Psychopathological Perspectives on Self-Experience. Amsterdam: John Benjamins, pp149-182.

Sheets-Johnstone, M. (2016). Insides and outsides. Interdisciplinary Perspectives on Animate Nature. Exeter, UK: Imprint Academic.

Shore, A. (2012). The Science of the Art of Psychotherapy. (Norton Series on Interpersonal Neurobiology). New York: WW. Norton

Sumathipala, A; Siribaddana, S; Abeysingha, MRN; de Silve, P; Dewey, M; Prince, M; and Mann, A.H. (2008). Cognitive-behaviour therapy v. structured care for medically unexplained symptoms: Randomized controlled trail. The British Journal of Psychiatry, 193, 51-59

Verhaak, P.F.M; Meijer, S.A; Visser, A.P; \& Wolters, G. (2006). Persistent presentation of medically unexplained symptoms in general practice. Family Practice, 23, (4) 414-420. 
Accepted Manuscript.

Book chapter (https://www.taylorfrancis.com/books/e/9781315159416/chapters/10.4324/9781315159416-19) published in "The

Routledge International Handbook of Embodies Perspectives in Psychotherapy: Approaches from Dance Movement and Body

Psychotherapies" (https://doi.org/10.4324/9781315159416), Routledge, 12 April 2019. 